ISSN 1112-9867

http://www.jfas.info

\title{
THE EFFECT OF ALLYL ISOTHIOCYANATE ON RNA
}

\author{
H. Habibi ${ }^{1, *}$ and M. Hashemi ${ }^{2}$ \\ ${ }^{1}$ Department of Genetics, Islamic Azad University, Tehran Medical Branch, Tehran, Iran \\ ${ }^{2}$ Mehrdad Hashemi, Department of Genetics, Islamic Azad University, Tehran Medical \\ Branch, Tehran, Iran
}

Published online: 05 June 2016

\begin{abstract}
Allyl isothiocyanate (AITC), finds in cruciferous vegetables. AITC shows bactericidal, fungicidal activities and anticancer activity by inhibition of cell proliferation, cell cycle arrest and apoptosis induction and tumor growth inhibition. In this study, the interaction of Allylisothiocyanate with yeast RNA in aqueous solution at physiological conditions using constant RNA concentration and various molar ratios of AITC/RNA complexes are examine. Fourier transform infrared (FTIR) and uv-visible difference spectroscopic methods are used to determine the AITC binding mode, binding constant and stability of RNA in AITC/RNA complexes in aqueous solution. Spectroscopic evidence shows that AITC interacts with RNA. The external binding of AITC to RNA with overall binding constant of $\mathrm{K}(\mathrm{AITC}-\mathrm{RNA})=7.207 \times 10^{6} \mathrm{M}^{-1}$ are also presented. RNA remains in A conformation upon AITC complexation.
\end{abstract}

Keywords: Allyl isothiocyanate; RNA; FTIR; UV-vis; binding constant; conformation.

Author Correspondence, e-mail: hedieh_habibi@yahoo.com

doi: http://dx.doi.org/10.4314/jfas.8vi2s.25 


\section{INTRODUCTION}

Allylisothiocyanate (3-isothiocyanato-1-propene or 2-propenyl isothiocyanate) is one the most common of Isothiocyanates which finds in cruciferous vegetables such as broccoli, cabbage, cauliflower, radish, wasabi and mustard (fig1.) (1-4).

\section{$\mathrm{CH} 2=\mathrm{CH}-\mathrm{CH} 2-\mathrm{N}=\mathrm{C}=\mathrm{S}$}

Fig.1. Chemical structure of Allyl isothiocyanate

Sinigrin which is predominant glucosinolate in cruciferous vegetables is hydrolyzed to Allyl isothiocyanate (AITC). The conversion of sinigrin to AITC catalyses by myrosinase or intestinal microflora in both animals and humans (figure 2.) (1, 4-7).

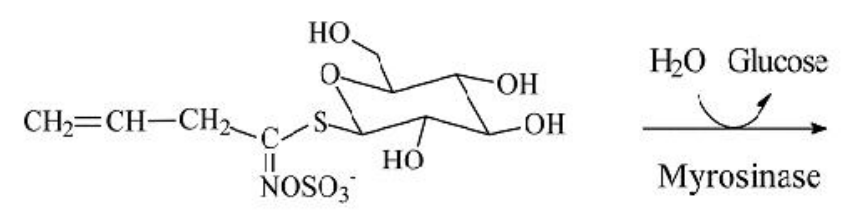

Sinigrin

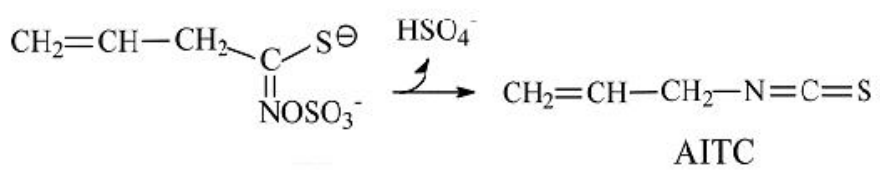

Fig.2. Conversion of sinigrin to Allyl isothiocyanate

Numerous studies suggest that AITC is a cancer chemo preventive agent. AITC shows bactericidal and fungicidal activities as well as anticancer activity with inhibition of cell proliferation, cell cycle arrest and apoptosis induction and tumor growth inhibition(5,8).

AITC causes apoptosis with arresting cell cycle at different stages of its progressions. Studies have shown that AITC arrested bladder cancer UM-UC-3 cells $(4,9,10)$, human cervical cancer Hela cells, human colorectal cancer HT29 cells in G2/M phase but arrested human leukemia HL60 cells in G1 phase(7).

In this study we have shown the interaction of AITC with RNA in aqueous solution at physiological conditions by using constant DNA concentration with the variety of molar ratios 
of AITC/RNA complexes. Fourier transform infrared (FTIR) and UV-visible spectroscopic were used to determine the AITC binding constant and stability of RNA in AITC/RNA complexes in aqueous solution.

\section{RESULTS AND DISCUSSION}

\subsection{FTIR spectra of AITC-RNA complexes}

The spectral changes (intensity and shifting) of several prominent RNA in-plane vibrations at $1698 \mathrm{~cm}^{-1}$ (Guanine, Uracil, mainly Guanine), $1650 \mathrm{~cm}^{-1}$ (Uracil, Guanine, Adenine, and Cytosine, mainly Uracil), $1610 \mathrm{~cm}^{-1}$ (mainly Adenine), $1488 \mathrm{~cm}^{-1} \quad$ (mainly Cytosine), 1241 $\mathrm{cm}^{-1}$ (PO2 asymmetric stretch) and $1080 \mathrm{~cm}^{-1}$ (PO2 symmetric) were monitored at different AITC-RNA molar ratios, and the results are shown in Figs. 3. These spectral changes determine that AITC binds to A-U, G-C or phosphate group (11-17).

If the loss of intensity has been occurred, it is related to RNA aggregation. While If AITC bind to RNA bases and the phosphate groups, major increase of intensity and shifting of those vibrations, will occur.

At $r=1 / 80$, increase of intensity was observed for asymmetric and symmetric phosphate bands but C, A, U had no significant changes upon AITC-RNA complexation. A significant decrease of intensity was observed for guanin. A band at 1607 shifted to 1609 , U band at 1649 shifted at $1650, \mathrm{C}$ band at 1485 shifted to 1482 , and asymmetric and symmetric phosphate band at 1238 and 1080 shifted to 1240 and 1082.

At $r=1 / 40$, decrease of intensity was observed for the symmetric phosphate but a significant increase of intensity was observed for U. But G, A, C and asymmetrc phosphate had not any significant changes upon complexation. The U band at 1649 shifted to 1650 , A band at 1607 shifted to $1606, \mathrm{C}$ band at 1485 shifted to 1488 , AP band at 1080 shifted to 1083 .

At $\mathrm{r}=1 / 20$, intensity of $\mathrm{G}, \mathrm{A}$ and $\mathrm{U}$ were decreased but the intensity of AP was increased. $\mathrm{P}$ and $\mathrm{C}$ had not any significant changes upon complexation. The A band at 1607 shifted to 1608 , The $\mathrm{C}$ band at 1485 shifted to 1488 , The AP band at 1080 shifted to 1079 .

At $r=1 / 10$, a major increasing of intensity for $G, U$ and $A$ was observed which shows the interaction of AITC with these bases. The intensity of C and AP were decreased 
insignificantly and P had not any significant changes upon complexation. The G band at 1694 shifted to 1697 , The $\mathrm{U}$ band at 1649 shifted to 1650 , The $\mathrm{C}$ band at 1485 shifted to 1483 , AP band at 1080 shifted to 1078 .

The intensities of the band at G(1694), U(1649), A(1607), AP(1080), P(1238) were decreased At higher concentration $(1 / 5,1 / 2,1 / 1)$. It can be resulted from RNA aggregation in the presence of AITC in aqueous solution. No major spectral changes were observed for C band at 1485 .

Evidence shows that AITC binds with guanine (N7), uracil (O2) and adenine (N7) residues. No major spectral changes were observed for cytosine at 1485 , that can be related to no major participation of cytosin in AITC-RNA binding.

In addition to spectral shifting of the PO2 stretching, the relative intensities of the asymmetric $\left(\mathrm{V}_{\mathrm{as}}\right)$ and symmetric $\left(\mathrm{V}_{\mathrm{s}}\right)$ vibrations were altered upon AITC interaction. Decreasing of $\mathrm{V}_{\mathrm{s}} / \mathrm{V}_{\mathrm{as}}$ from 2.04 (free RNA) to 1.46 (AITC-RNA complexes), can be related to AITC-PO2 interaction.

\subsection{RNA Conformation}

RNA often is in A-conformation. There are some marker bands at $810-862 \mathrm{~cm}^{-1}$ (ribose phosphate), $1241 \mathrm{~cm}^{-1}$ (assymetric phosphate) and $1698 \mathrm{~cm}^{-1}$ (guanine) for showing the A-conformation of RNA. The lack of major shifting of these marker bands is indicative of RNA remaining in A-conformation upon AITC-RNA complexation. 


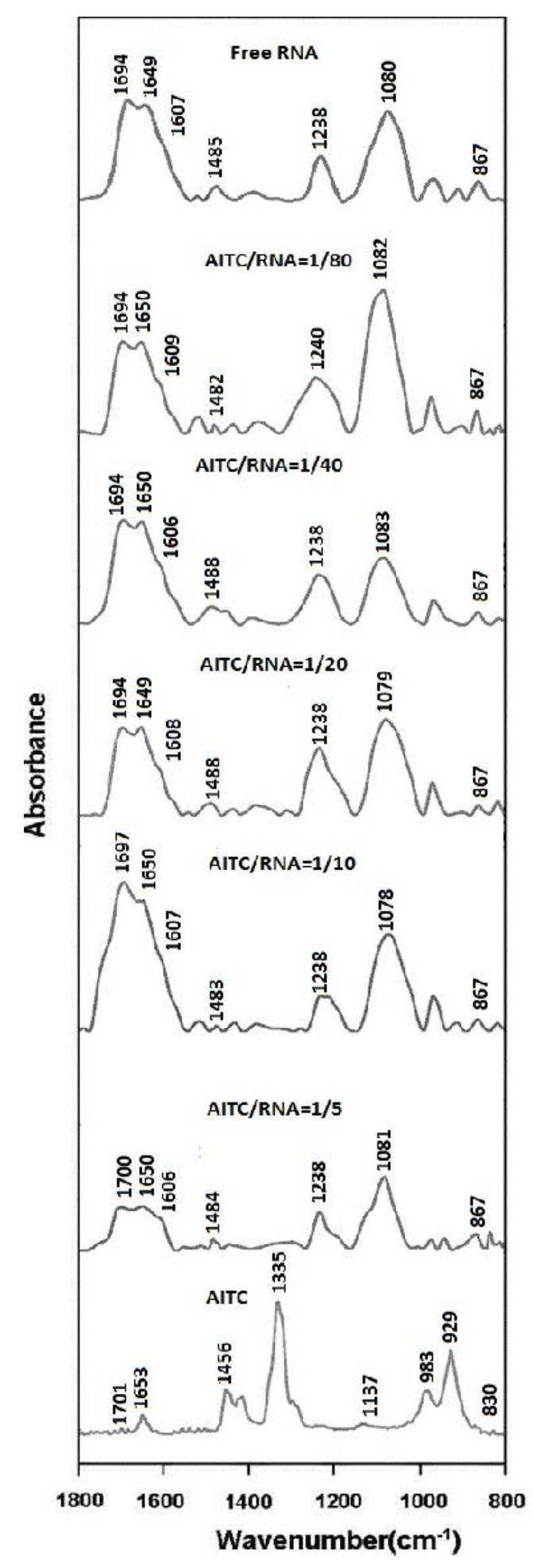

Fig.3. FTIR spectra and difference FTIR spectra [(RNA solution + AITC)-RNA solution] of free yeast RNA and AITC complexes at different molar ratios in the region of $1800-800 \mathrm{~cm}^{-1}$ in aqueous solution 


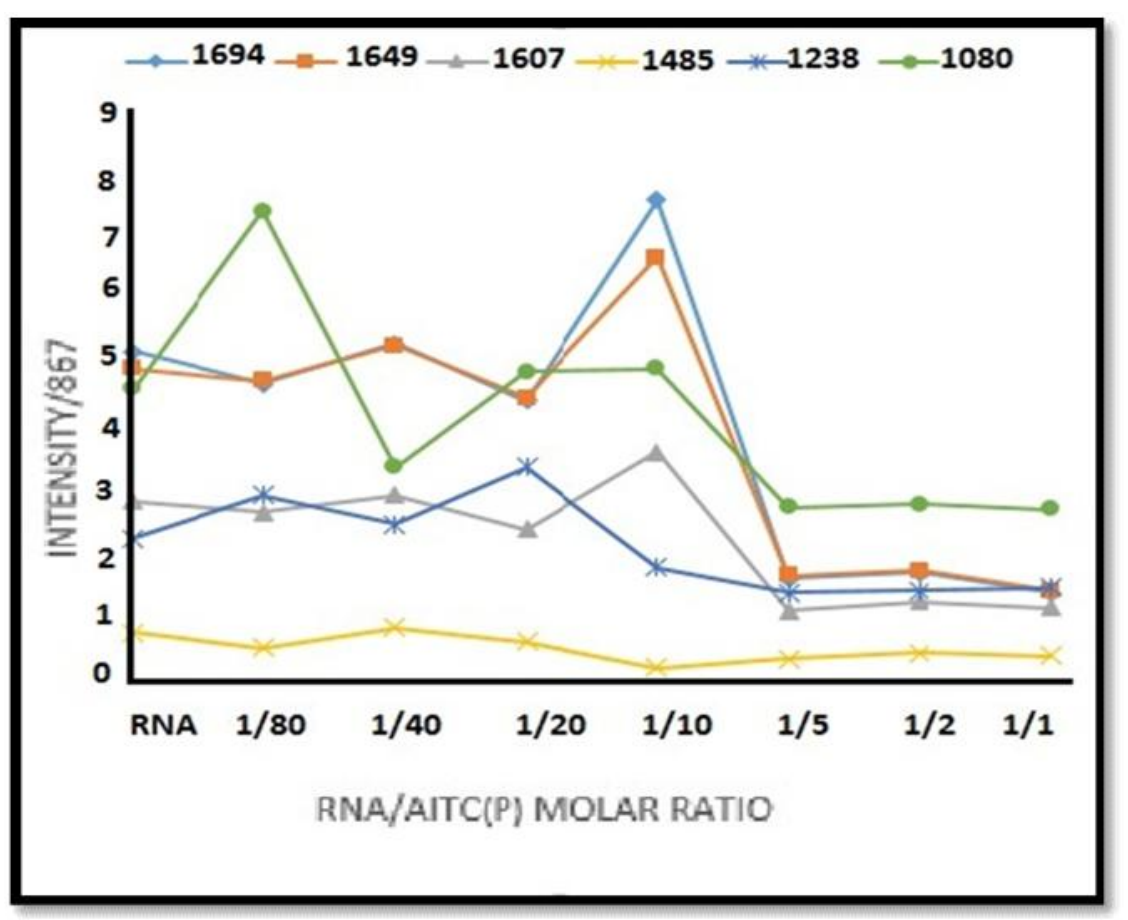

Fig.4. Intensity ratio variations for AITC-RNA complexes

\subsection{Absorption spectra of AITC-RNA complexes}

The AITC binding constants were determined as described in experimental section (UV-Vis spectroscopy)(18, 19).

The concentrations of complexed ligand was determined by subtracting the absorbance of free RNA at $260 \mathrm{~nm}$ from the complexed RNA and concentration of free ligand was determined by subtracting of complexed ligand from total ligand which were used for the experiment. Our data of $1 /[$ ligand complexed] almost proportionally increased as a function of $1 /$ [free ligand] . The double - reciprocal plot of $1 /\left(\mathrm{A}-\mathrm{A}_{0}\right)$ versus $1 /$ (ligand concentration) is linear, and the binding constant $(\mathrm{K})$ can be estimated from the ratio of intercept to the slope (Figure 5).

$\mathrm{A}_{0}$ is the initial absorbance of the free RNA at $260 \mathrm{~nm}$ and $\mathrm{A}$ is the recorded absorbance of RNA in presence of different AITC concentrations.

The overall binding constant is estimated to be $\mathrm{K}=7.207 \times 10^{6} \mathrm{M}^{-1}$ for AITC-RNA complexes which shows that AITC binds RNA strongly. Similar association constants were reported for RNA complexes with Arsenic Trioxide(20), ß-carboline alkaloids(21), glycyrrhizin and glycyrrhetinic(22).

If the intensity of ligand characteristic UV-Vis band increases, ligand interacts with RNA 
surface. In this study, a major increasing in intensity of UV-Vis bands characteristic $258 \mathrm{~nm}$ of AITC is indicative of hyper chromosity. This hyper chromic shift, indicates interacting of AITC with RNA(Fig.6.)(23).

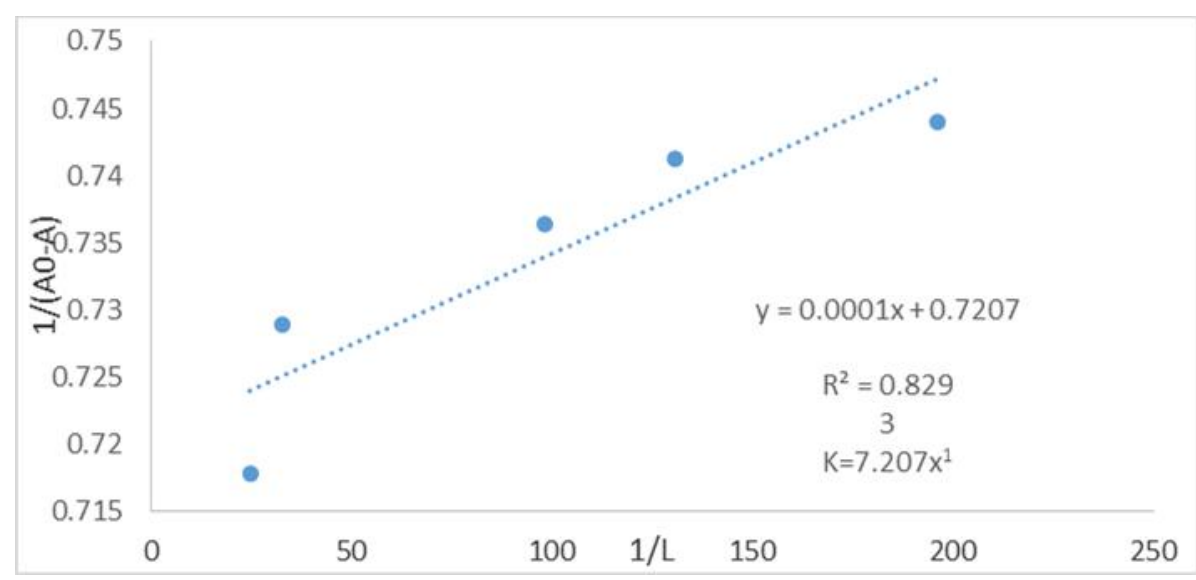

Fig.5. The plot of $1 /\left(\mathrm{A}-\mathrm{A}_{0}\right)$ versus $1 / \mathrm{L}$ for RNA and their ligand complexes

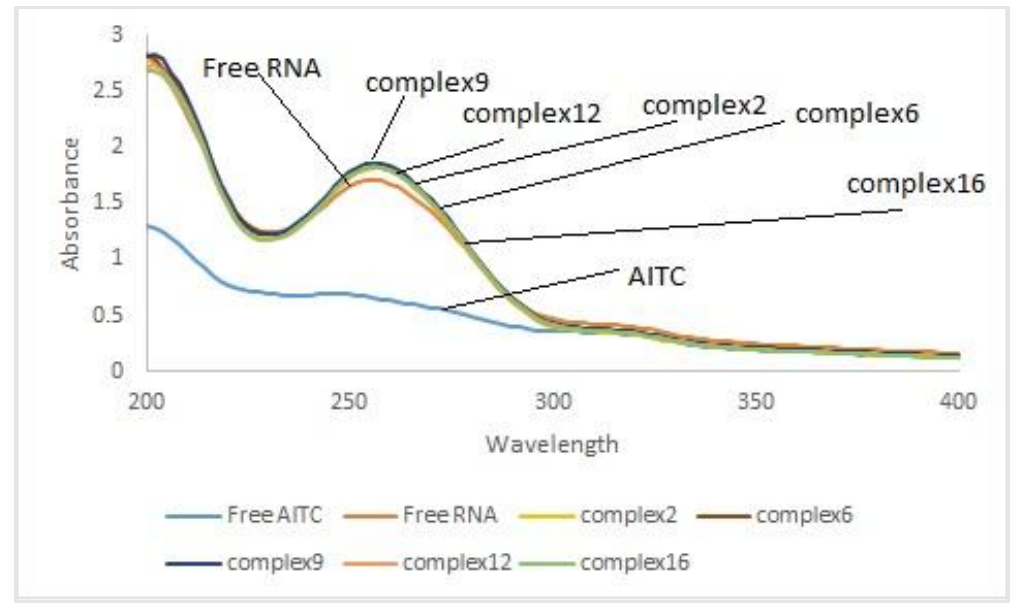

Fig.6. UV-Vis spectra characteristics of AITC and AITC/RNA complex

\section{EXPERIMENTAL}

\subsection{Materials}

Yeast Baker RNA sodium salt and Allyl isothiocyanate were purchased from Sigma chemical Co.

\subsection{Preparation of stock Solutions}

Sodium-RNA $(5 \mathrm{mg} / \mathrm{ml})$ was dissolved in distilled water at $5^{\circ} \mathrm{C}$ for $24 \mathrm{~h}$ with occasional stirring to ensure the formation of homogeneous solutions. The solutions of AITC at different 
concentrations $(12.5,6.25,2.5,1.25,0.625,0.3125,0.1562)$ were prepared in distilled water-ethanol(50/50\%) and added dropwise to the RNA solutions with constant stirring to give the desired AITC/RNA (p) molar ratios of 1/80 to 1 with a final RNA concentration of $6.25 \mathrm{mM}$ (phosphate) . The PH of the solutions was adjusted between 6 and 7 with $\mathrm{NaOH}$ solution(24).

It should be noted that ethanol at $75 \%$ concentration facilitates conformational transition, where as at lower concentrations ethanol content (such as 50/50\% ethanol/water used here) does not effect RNA conformation $(25,26)$.

\subsection{FTIR Spectroscopy}

In this study, Thermo Nicolet spectrometer(Magna550) with a liquid- nitrogen- cooled $\mathrm{HgCdTe}(\mathrm{MCT})$ detector was used to record the infrared spectra. The spectra of AITC and RNA/AITC complexes were acquired using ZnSe cell with resolution of $4 \mathrm{~cm}^{-1}$ and 100 scans. OMNIC software which supplied by manufacturer of spectrophotometer was used to detect and treat the spectra. The difference spectra [(RNA solution + AITC) - (RNA solution)] were produced, using a sharp bands at $867 \mathrm{~cm}^{-1}$ for RNA as internal references. This band is due to the sugar $\mathrm{C}-\mathrm{C}$ and sugar phosphate stretching modes and does not show any spectral changes upon AITC/RNA interaction. These intensity ratios were used to detect the AITC binding to RNA bases or the backbone phosphate groups. Similar intensity variations have been used to determine the AITC binding to RNA bases and backbone phosphate groups(27-30).

\subsection{Absorption spectroscopy}

The absorption spectra were recorded on a LTD model T90 UV Vis spectrometer PG Instruments and Quartz cuvettes of $1 \mathrm{~cm}$ were used. Spectra manager software was used for collecting the spectra and Analysis spectrum software was used for spectra analysing. In this study UV-visible spectroscopy was used to determine the binding constant of AITC-RNA complex.

The concentration of RNA was remained on $0.51 \mathrm{mM}$, while various AITC concentration of $2.04 \times 10^{-4} \mathrm{M}$ to $1.02 \times 10^{-6} \mathrm{M}$ were used. 


\section{CONCLUSION}

Allyl isothiocyanate binds RNA with external binding. The overall binding constant of AITC-RNA is $\mathrm{K}=7.207 \times 10^{6} \mathrm{M}^{-1}$ that indicate strong ligand-RNA interaction. Spectroscopy evidence shows that AITC binds with Guanine(N7), Uracil(O2) and Adenine(N7) residues. AITC also binds externally with phosphate-sugar backbone of RNA. RNA remains in A-form in all concentration.

\section{ACKNOWLEDGEMENTS}

We thank Islamic Azad University, Tehran medical sciences Branch for support of this work.

\section{REFERENCES}

[1] Hwang E-S, Lee HJ. Allyl isothiocyanate and its N-acetylcysteine conjugate suppress metastasis via inhibition of invasion, migration, and matrix metalloproteinase-2/-9 activities in SK-Hep1 human hepatoma cells. Exp Biol Med. 2006;231(4):421-30.

[2] Hwang E-S, Kim GH. Allyl isothiocyanate influences cell adhesion, migration and metalloproteinase gene expression in SK-Hep1 cells. Exp Biol Med. 2009;234(1):105-11.

[3] Zhang Y. The molecular basis that unifies the metabolism, cellular uptake and chemopreventive activities of dietary isothiocyanates. Carcinogenesis. 2012;33(1):2-9.

[4] Bhattacharya A, Li Y, Wade KL, Paonessa JD, Fahey JW, Zhang Y. Allyl isothiocyanate-rich mustard seed powder inhibits bladder cancer growth and muscle invasion. Carcinogenesis. 2010;31(12):2105-10.

[5] Zhang Y. Allyl isothiocyanate as a cancer chemopreventive phytochemical. Mol. Nutr. Food Res. 2010;54(1):127-35.

[6] Smith TK, Mithen R, Johnson IT. Effects of Brassica vegetable juice on the induction of apoptosis and aberrant crypt foci in rat colonic mucosal crypts in vivo. Carcinogenesis. 2003;24(3):491-5.

[7] Zhang Y, Tang L, Gonzalez V. Selected isothiocyanates rapidly induce growth inhibition of cancer cells. Mol. Cancer Ther. 2003;2(10):1045-52.

[8] Okulicz M. Multidirectional time-dependent effect of sinigrin and allyl isothiocyanate on 
metabolic parameters in rats. Plant Foods Hum Nutr. 2010;65(3):217-24.

[9] Smith TK, Lund EK, Parker ML, Clarke RG, Johnson IT. Allyl-isothiocyanate causes mitotic block, loss of cell adhesion and disrupted cytoskeletal structure in HT29 cells. Carcinogenesis. 2004;25(8):1409-15.

[10] Bhattacharya A, Tang L, Li Y, Geng F, Paonessa JD, Chen SC, et al. Inhibition of bladder cancer development by allyl isothiocyanate. Carcinogenesis. 2010;31(2):281-6.

[11] DiRico DE, Keller PB, Hartman KA. The infrared spectrum and structure of the type I complex of silver and DNA. Nucleic Acids Res. 1985;13(1):251-60.

[12] Loprete D, Hartman K. Conditions for the stability of the B, C, and Z structural forms of poly ( $\mathrm{dG}-\mathrm{dC})$ in the presence of lithium, potassium, magnesium, calcium, and zinc cations. Biochemistry. 1993;32(15):4077-82.

[13] Alex S, Dupuis P. FT-IR and Raman investigation of cadmium binding by DNA. Inorg. Chim. Acta. 1989;157(2):271-81.

[14] Starikov E, Semenov M, Gasan A. Evidential study of correlated events in biochemistry: physicochemical mechanisms of nucleic acid hydration as revealed by factor analysis. Biopolymers. 1991;31(3):255-73.

[15] Taillandier E, Liquier J. [16] Infrared spectroscopy of DNA. Methods Enzymol. 1992;211:307-35.

[16] Andrushchenko V, Leonenko Z, Cramb D, van de Sande H, Wieser H. Vibrational $\mathrm{CD}$ (VCD) and atomic force microscopy (AFM) study of DNA interaction with $\mathrm{Cr} 3+$ ions: VCD and AFM evidence of DNA condensation. Biopolymers. 2002;61(4):243-60.

[17] Dovbeshko G, Chegel V, Gridina NY, Repnytska O, Shirshov Y, Tryndiak V, et al. Surface enhanced IR absorption of nucleic acids from tumor cells: FTIR reflectance study. Biopolymers. 2002;67(6):470-86.

[18] Klotz IM, Hunston DL. Properties of graphical representations of multiple classes of binding sites. Biochemistry. 1971;10(16):3065-9.

[19] Zhong W, Wang Y, Yu JS, Liang Y, Ni K, Tu S. The interaction of human serum albumin with a novel antidiabetic agent—SU-118. J. Pharm. Sci. 2004;93(4):1039-46.

[20] Nafisi S, Sobhanmanesh A, Alimoghaddam K, Ghavamzadeh A, Tajmir-Riahi H-A. 
Interaction of arsenic trioxide As2O3 with DNA and RNA. DNA Cell Biol. 2005;24(10):634-40.

[21] Nafisi S, Malekabady ZM, Khalilzadeh MA. Interaction of $\beta$-carboline alkaloids with RNA. DNA Cell Biol. 2010;29(12):753-61.

[22] Nafisi S, Manouchehri F, Bonsaii M. Study on the interaction of glycyrrhizin and glycyrrhetinic acid with RNA. J. Photochem. Photobiol. B, Biol. 2012;111:27-34.

[23] Kanakis C, Nafisi S, Rajabi M, Shadaloi A, Tarantilis P, Polissiou M, et al. Structural analysis of DNA and RNA interactions with antioxidant flavonoids. j. spectrosc. 2009;23(1):29-43.

[24] Vijayalakshmi R, Kanthimathi M, Subramanian V, Nair BU. DNA cleavage by a chromium (III) complex. Biochem. Biophys. Res. Commun. 2000;271(3):731-4.

[25] Potaman V, Bannikov YA, Shlyachtenko L. Sedimentation of DNA in ethanol-water solutions within the interval of $\mathrm{B} \rightarrow$ A transition. Nucleic Acids Res. 1980;8(3):635-42.

[26] Nejedlý K, Chládková J, Vorlíčková M, Hrabcová I, Kypr J. Mapping the BA conformational transition along plasmid DNA. Nucleic Acids Res. 2005;33(1):e5-e.

[27] Arakawa H, Ahmad R, Naoui M, Tajmir-Riahi H-A. A Comparative Study of Calf Thymus DNA Binding to $\mathrm{Cr}$ (III) and $\mathrm{Cr}$ (VI) Ions Evidence for the guanine N-7-CHROMIUM-PHOSPHATE chelate formation. J. Biol. Chem. 2000;275(14):10150-3.

[28] Neault J, Tajmir-Riahi H. Structural analysis of DNA-chlorophyll complexes by Fourier transform infrared difference spectroscopy. Biophys. J. 1999;76(4):2177-82.

[29] Kanakis C, Tarantilis P, Polissiou M, Diamantoglou S, Tajmir-Riahi H. DNA interaction with naturally occurring antioxidant flavonoids quercetin, kaempferol, and delphinidin. J Biomol Struct Dyn. 2005;22(6):719-24.

[30] Kanakis C, Tarantilis P, Polissiou M, Diamantoglou S, Tajmir-Riahi H. An overview of DNA and RNA bindings to antioxidant flavonoids. Cell Biochem Biophys. 2007;49(1):29-36. 


\section{How to cite this article:}

Habibi $\mathrm{H}$ and Hashemi M. The effect of allyl isothiocyanate on RNA. J. Fundam. Appl. Sci., 2016, 8(2S), 301-312. 